\title{
Nash rationalization of collective choice over lotteries
}

\author{
Thomas Demuynck $^{1}$, Luc Lauwers ${ }^{2}$ * \\ ${ }^{1}$ Sherppa, Ghent University, \\ Tweekerkenstraat 2, 9000 Gent, Belgium; \\ tel: +32 (0)9 26495 09; fax +32(0)9 2648996 \\ e-mail: Thomas.Demuynck@ugent.be \\ ${ }^{2}$ Center for Economic Studies, K.U.Leuven, \\ Naamsestraat 69, 3000 Leuven, Belgium; \\ tel: +32 (0)16 3268 09; fax: +32 (0)16 326796 \\ e-mail: Luc.Lauwers@econ.kuleuven.be
}

First version: April, 2005. This version: July 14, 2008.

\begin{abstract}
To test the joint hypothesis that players in a noncooperative game (allowing mixtures over pure strategies) consult an independent preference relation and select a Nash equilibrium, it suffices to study the reaction of the revealed collective choice upon changes in the space of strategies available to the players. The joint hypothesis is supported if the revealed choices satisfy an extended version of Richter's congruence axiom together with a contractionexpansion axiom that models the noncooperative behavior. In addition, we provide sufficient and necessary conditions for a binary relation to have an independent ordering extension, and for individual choices over lotteries to be rationalizable by an independent preference relation.
\end{abstract}

Keywords and Phrases: independence condition, binary extensions, rationalizability, Nash equilibrium with mixed strategies.

JEL Classification Numbers: C72, C92.

${ }^{*}$ We thank two anonymous referees, an associate editor, and Dirk Van de gaer for helpful comments. Thomas Demuynck, aspirant Fonds voor Wetenschappelijk Onderzoek Vlaanderen (FWO). 


\section{Introduction}

A recent track of research seeks to identify the testable implications of various theories of multi-agent decision making. Along these lines we set up a test to verify whether players have independent preference relations and select a Nash equilibrium. Let us start the exposition with an example.

Consider a two person game in normal form. Each player has two pure strategies: $U(\mathrm{p})$ and $D$ (own) for player $1, L$ (eft) and $R$ (ight) for player 2 . Each player is informed about the meaning of a mixture over pure strategies and about the payoff such a mixture generates. Let $x$ (resp. $y$ ) denote the weight attached to the pure strategy $U$ (resp. $L$ ). The players may select the mixture

$$
x \times U+(1-x) \times D \quad \text { and } \quad y \times L+(1-y) \times R,
$$

with $x$ and $y$ in the closed interval $[0,1]$, and communicate the selected value of $x$ (resp. $y$ ) to the experimental designer. ${ }^{1}$ In this setup, we observe the values $x=0.4$ and $y=0.3$. Then, a second experiment is executed. For player 1, the set of pure strategies $\{U, D\}$, is modified to $\left\{U, D^{\prime}\right\}$ with

$$
D^{\prime}=0.4 \times U+0.6 \times D .
$$

For player 2 , the set $\{L, R\}$ is modified to $\left\{L^{\prime}, R\right\}$ with

$$
L^{\prime}=0.42 \times L+0.58 \times R \text {. }
$$

The lottery $x^{\prime} \times U+\left(1-x^{\prime}\right) \times D^{\prime}$ coincides with $\left(x^{\prime}+\left(1-x^{\prime}\right) 0.4\right) \times U+\left(1-x^{\prime}\right) 0.6 \times D$ and the lottery $y^{\prime} \times L^{\prime}+\left(1-y^{\prime}\right) \times R$ coincides with $0.42 y^{\prime} \times L+\left(1-0.42 y^{\prime}\right) \times R$. Given these sets of pure strategies, player 1 selects $0.4 U+0.6 \mathrm{D}$ (i.e. $x^{\prime}=0$ ) and player 2 selects $0.42 L+0.58 R$ (i.e. $y^{\prime}=1$ ). Similar experiments generate the following data:

\begin{tabular}{llll} 
pure strategies & & $\longmapsto$ & \multicolumn{2}{c}{ selected mixtures } \\
\hline player 1 & player 2 & player 1 & player 2 \\
$\{U, D\}$ & $\{L, R\}$ & $.4 U+.6 D$ & $.3 L+.7 R$ \\
$\left\{U, D^{\prime}=.4 U+.6 D\right\}$ & $\left\{L, R^{\prime}=.42 L+.58 R\right\}$ & $.4 U+.6 D$ & $.42 L+.58 R$ \\
$\left\{U^{\prime \prime}=.5 U+.5 D, D\right\}$ & $\left\{L^{\prime \prime}=R^{\prime \prime}=.5 L+.5 R\right\}$ & $.5 U+.5 D$ & $.5 L+.5 R$
\end{tabular}

TABLE 1: OBSERVED DATA.

The following question arises. Given such data, is it possible to check whether or not these players are rational in the sense that they optimize with respect to an independent preference relation and select a Nash equilibrium? Section 4 returns to this example and indicates that the above data are not Nash rationalizable. The remaining part of the introduction positions this research in the literature and introduces our main results.

\footnotetext{
${ }^{1}$ Sopher and Narramore (2000) carry out an experiment to test consistency and (in)transitivity of individual choice over lotteries and mixtures of lotteries. In the spirit of their questionnaire, we propose the players to select from a menu of mixtures over pure strategies.
} 
Many theories on behavior start from assumptions on the individual preference relation over the feasible set of alternatives (e.g. transitivity, completeness). As soon as one accepts that binary relations are not observable while actual choices are observable; it is important to test whether the actual choices support or reject the assumptions. This issue has been discussed by, among others, Arrow (1959), and Sen (1971).

There are at least two ways to tackle this problem. One approach (Sen, 1971) studies how the selection reacts upon particular changes in the set of feasible alternatives. Obviously, if the individual consults a transitive and complete preference relation, then he should not reconsider his choice when the choice set shrinks while his selected alternative remains feasible. Analogously, when he selects the same alternative from two different choice sets, then he should select again this alternative from the union of these two choice sets. As such, the hypothesis of revealed preference becomes testable. A second approach is offered through the theory of revealed preferences. If an alternative is chosen from a set, then it is top ranked in this choice set according to the revealed preference relation. The transitive closure of this revealed preference relation is called the indirect revealed preference relation. Richter's (1966) congruence axiom provides necessary and sufficient conditions for a choice function to be rationalizable: if an alternative $a$ is indirectly revealed preferred to $b$, then $b$ should not be strictly revealed preferred to $a$.

Sprumont (2000) extends the problem of rationalizability to situations involving different and interacting individuals. He defines a joint choice function to be Nash rationalizable if there exists a profile of complete and transitive preference relations over the sets of actions, so that the observed outcomes coincide with the Nash equilibria based upon these preferences. In the spirit of Sen's approach, he characterizes Nash rationalization through the combination of an expansion and a contraction property. Ray and Zhou (2001) perform a similar study for subgame perfect Nash equilibria.

We extend one of the results of Sprumont (2000) and tackle the Nash rationalizability of collective choice when individuals have a menu of mixtures at their disposal (each mixture defines a probability distribution over the set of pure strategies). For example, Table 1 might result from an experiment. Following the tradition in game theory, we interpret the rational behavior of a player in terms of expected utility maximization. In particular, besides completeness and transitivity we impose an independence demand upon the preference relations of the (rational) players. This independence condition states that the relationships between two lotteries (over the set of pure strategies profiles) are not affected when they are mixed in the same way with a third lottery. Myerson (1997, p11) discusses the strength of the independence axiom in the expected utility maximization theorem. In addition, he indicates some of the difficulties that arise in decision theory when independence is dropped. As a matter of fact, Clark (2000) introduces a 'revealed Archimedean axiom' (a continuity axiom) to capture the difference between rationalization by an independent ordering and maximizing expected utility.

Furthermore, in contrast to Sprumont (2000), we follow the track of revealed preferences. If only one individual is involved, it is sufficient to check the transitive closure of the revealed 
preference relation. The present setting with more than one individual, however, is more demanding. We modify Richter's axiom and require that the 'transitive and independent' closure of the revealed preference relation does not conflict with the strict revealed preference relation. Besides that, we need an axiom that connects the individual behavior to the collective behavior. We assume that a strategy profiles belongs to the collective choice if each player keeps his selected strategy when he is assured that he is the only player allowed to deviate. We refer to this condition as the collective choice being noncooperative. Later on, we will argue that this condition has some flavor of an expansion-contraction axiom. Our main result reads (see Theorem 3 in Section 4 for the exact formulation):

Theorem. A collective choice correspondence is Nash rationalizable if and only if it is noncooperative and satisfies the modified version of Richter's congruence axiom.

Let us highlight two intermediate results towards this theorem. First, we need a condition that is strong enough to guarantee that a binary relation extends to a transitive, complete, and independent relation. Here, we learn from Suzumura (1976), who showed that consistency is sufficient and necessary for a relation to have an ordering extension. We shift Suzumura's result to a setup involving choices over lotteries, and we use the term 'lottery-consistency' as a reference. Second, we study the behavior of a single individual choosing from a set of lotteries. Here, we show that the extended version of Richter's congruence axiom - restricted to one player - is sufficient and necessary for the individual choice function to be rationalizable by an independent ordering. ${ }^{2}$ Then, we broaden the setup from one individual to a finite number of interacting players. We apply the axiom of noncooperation behavior and conclude the above theorem. ${ }^{3}$

This theorem can be used in an experimental setting to test whether players have independent preference relations and select a Nash equilibrium. First, each player is told the structure of the game: the number of players, the sets of pure strategies, the concept of a mixture (and its interpretation as a probability distribution over the set of pure strategies), and the payoff function. Subsequently, each individual is informed about the menus available to all of the players, and is asked to choose from 'his' menu of mixtures. The size of such a menu is either finite (e.g. MacDonald and Wall, 1989; Conlisk, 1989; Oliver, 2003) or infinite (Sopher and Narramore, 2000; Shachat, 2002). Once each player has independently chosen a mixture from his menu, the experimenter determines the resulting lottery over the pure strategy profiles and the corresponding outcome.

The assumption that outcomes from mixed strategies are observable is crucial and we recognize the intrinsic difficulties. The problem is especially manifest if observations only encompass the pure strategy outcomes that result from the mixed strategy profile. For these situations, the one shot Nash equilibrium in mixed strategies is a rather unappealing equilibrium concept. However, in cases where the experimenter controls the outcomes,

\footnotetext{
${ }^{2}$ Section 3 discusses similar results obtained by Clark (1993, Thm 3) and by Kim (1996, Thm 3.1).

${ }^{3}$ Galambos (2005) considers a related set-up where the strategy space is restricted to consists only of pure strategies. He introduces an analogous condition (I-congruence) for the Nash rationalizability. See section 4 for a discussion.
} 
one may resort to the following setup. Each individual is asked to select a $t$-tuple of pure strategies from his menu. Then, the experimenter computes for each individual the corresponding probability distribution over the set of pure strategies, and computes - on the basis of the product distribution - the corresponding outcome of the game. This setup certainly avoids the problem of correlated distributions in case the game (and the one-shot Nash equilibrium) is repeated $t$ times. Furthermore, taking $t$ large enough each lottery can be approximated as accurately as desired.

In order to check whether individuals play a Nash equilibrium, we propose to proceed in two stages. In a first experiment, the players are screened according to whether they maximize with respect to an independent preference relation. This can be done, for instance, through some Allais-paradox test (e.g. Conlisk, 1989; Oliver, 2003). This step filters out those individuals who violate the expected utility criterion. In the second step, one confronts the remaining players with a noncooperative game. As such, one can judge on the basis of observations whether in mixed strategies the Nash criterion is rejected or supported.

The next section introduces the notation and studies binary relations and their independent and transitive extensions. Section 3 introduces the concept of lottery-consistency as a test for the rational behavior of an individual choosing over lotteries, and discusses related results of Clark (1993) and Kim (1996). Section 4 extends the notation to collective choice and proves the main result. Here, we also return to the data in Table 1. Section 5 links our result to the analysis of Sprumont (2000). Finally, in Section 6 we demonstrate the independency of the key axioms (noncooperation and congruence).

\section{Independent ordering extensions}

This section establishes the notation, introduces the concept of independence of a binary relation, and provides conditions for a relation to have an independent ordering extension.

Let $H \subset \mathbb{R}^{n}$ be the hyperplane of $n$-vectors the coordinates of which add up to 1 , and let $\Delta=\Delta^{n} \subset H$ be the $(n-1)$-dimensional simplex. ${ }^{4}$ An element $x=\left(x_{1}, x_{2}, \ldots, x_{n}\right)$ in $\Delta$ is an $n$-tuple of nonnegative real numbers adding up to 1 , and is called a lottery. The $i$-th coordinate $x_{i}$ of the lottery $x$ gives the probability that state $i$ occurs.

Throughout, the set $D$ refers to either $\Delta$ or $H$. A binary relation $R$ in the set $D$ is a subset of the cartesian product $D \times D$. The symmetric component $R \cap R^{-1}$ is denoted by $I$, the asymmetric part $R \backslash I$ by $P$, and the non-comparable part $D \times D \backslash\left(R \cup R^{-1}\right)$ by $N$. For the binary relation $R^{\prime}$ we denote these induced relations by $I^{\prime}, P^{\prime}, N^{\prime}$; for $R^{*}$ we use $I^{*}$, $P^{*}, N^{*}$; etc. A reflexive and transitive relation is said to be a quasi-ordering. A complete quasi-ordering is said to be an ordering. The binary relation $R^{\prime}$ in $D$ extends the relation $R$ if $R \subset R^{\prime}$ and $P \subset P^{\prime}$.

\footnotetext{
${ }^{4}$ For two sets $A$ and $B$, we write $A \subset B$ if each element of $A$ belongs to $B$. The combination $A \subset B$ and $B \subset A$ is summarized as $A=B$.
} 
Next, we introduce the notion of independence. This condition studies the behavior of a binary relation on compound vectors. For $x$ and $y$ in $H$ and for $\alpha$ a nonnegative real number, the vector $[\alpha, x, y]$ denotes the linear combination $\alpha x+(1-\alpha) y$. For $\alpha$ in between 0 and 1 , the compound vector $[\alpha, x, y]$ is a convex combination of $x$ and $y$. And, for $\alpha>1$ the compound vector is a point on the ray starting in $y$ and going through $x$ and does not belong to the closed interval $[x, y]$.

A relation $R$ in $D$ is said to be independent if for each pair in $R$ the composition with a third vector in $D$ preserves the initial relationships. Formally, $R$ is independent if for each $x, y$, and $z$ in $D$, we have

$$
\text { if }(x, y) \in R, \alpha \geq 0,[\alpha, x, z] \in D \text {, and }[\alpha, y, z] \in D \text {; then }([\alpha, x, z],[\alpha, y, z]) \in R \text {. }
$$

This condition implies the reflexivity of $R$ (put $\alpha=0$ ). Observe that $\alpha$ is allowed to take values larger than 1 . As a consequence of this, an independent relation satisfies the 'strict' version of condition (1):

$$
\text { if }(x, y) \in P, \alpha>0,[\alpha, x, z] \in D \text {, and }[\alpha, y, z] \in D \text {; then }([\alpha, x, z],[\alpha, y, z]) \in P \text {. }
$$

Indeed, let us assume that $(x, y) \in P$ and $\alpha>0$, while $([\alpha, x, z],[\alpha, y, z]) \notin P$. Since $R$ is independent and $(x, y) \in P \subset R$, it follows that $([\alpha, x, z],[\alpha, y, z]) \in R$. The assumption $([\alpha, x, z],[\alpha, y, z]) \notin P$ implies that $([\alpha, y, z],[\alpha, x, z]) \in R$. Therefore,

$$
(y, x)=\left(\left[\frac{1}{\alpha},[\alpha, y, z], z\right],\left[\frac{1}{\alpha},[\alpha, x, z], z\right]\right) \in R .
$$

A contradiction is obtained: $(y, x) \in R$ and $(x, y) \in P$. Note that $\alpha$ and $1 / \alpha$ simultaneously occur (one of these values is larger than 1 ).

In case $R$ happens to be a complete binary relation, a similar argument implies that $R$ is independent if and only if $R$ is reflexive and for each $x, y, z$ in $D$, and each $\alpha$, we have

$$
\text { if }(x, y) \in R \text { (resp. } P) \text { and } 0<\alpha \leq 1 \text {, then }([\alpha, x, z],[\alpha, y, z]) \in R(\operatorname{resp} . P) \text {. }
$$

Obviously, condition (1) entails condition (2). Let us check that (2) implies (1). Suppose the antecedent clause of (1) holds, and let $\alpha>1$. Then, the opposite conclusion - in the assumption that $R$ is complete - reads: "( $[\alpha, y, z],[\alpha, x, z]) \in P$ ". Again, we obtain a contradiction: $(y, x) \in P$ while $(x, y) \in R$.

Condition (2) only considers convex combinations and is therefore, in the present setting, perhaps a more natural property.

There is an obvious relationship between the class of independent orderings on $H$ and the class of independent orderings on $\Delta$. If $R$ is an independent ordering on $H$, then its restriction to $\Delta$ is an independent ordering on $\Delta$. The next lemma looks at the reverse relationship.

Lemma 1. An independent, transitive, and complete relation $R$ in $\Delta$, uniquely extends to an independent, transitive, and complete relation $R^{\prime}$ in $H$. 
Proof. Let $x$ and $y$ belong to $H$. Let $z$ be an element in the interior of $\Delta$. Choose $\alpha>0$ sufficiently close to 0 , such that $x^{\prime}=[\alpha, x, z]$ and $y^{\prime}=[\alpha, y, z]$ belong to $\Delta$. Let the ordering $R^{\prime}$ on $\{x, y\}$ agree with the ordering $R$ on $\left\{x^{\prime}, y^{\prime}\right\}$. The ordering $R^{\prime}$ on $\{x, y\}$ does not depend upon the choice of $z$ and $\alpha$. We show this by contradiction. Let $x^{\prime \prime}=[\beta, x, u] \in \Delta$ and $y^{\prime \prime}=[\beta, y, u] \in \Delta$ and assume that $\left(x^{\prime}, y^{\prime}\right) \in R$ while $\left(y^{\prime \prime}, x^{\prime \prime}\right) \in P$. By independence, we have

$$
(\underbrace{\left[\frac{\beta}{\alpha+\beta}, x^{\prime}, y^{\prime \prime}\right]}_{v_{1}}, \underbrace{\left[\frac{\beta}{\alpha+\beta}, y^{\prime}, y^{\prime \prime}\right]}_{v_{2}}) \in R \text { and }(\underbrace{\left[\frac{\alpha}{\alpha+\beta}, y^{\prime \prime}, y^{\prime}\right]}_{v_{3}}, \underbrace{\left[\frac{\alpha}{\alpha+\beta}, x^{\prime \prime}, y^{\prime}\right]}_{v_{4}}) \in P .
$$

Observe that $v_{2}$ and $v_{3}$ coincide. Transitivity of $R$ implies that $\left(v_{1}, v_{4}\right) \in P$. One can write $v_{1}$ and $v_{4}$ in terms of $x, y, z$, and $u$ and verify that $v_{1}=v_{4}$. Hence, we obtain $\left(v_{1}, v_{1}\right) \in P$. This contradicts the definition of the asymmetric component $P$ of the relation $R$. Therefore, $R^{\prime}$ is well defined. Transitivity and independence of $R^{\prime}$ follows from the definition of $R^{\prime}$ in combination with the transitivity and independence of $R$.

Finally, we show that the extension $R^{\prime}$ is unique. Let the independent, transitive, and complete relations $R^{\prime}$ and $R^{\prime \prime}$ extend $R$. Let $x$ and $y$ belong to $H$. Let $z$ be an element in the interior of $\Delta$. Choose $\alpha>0$ sufficiently close to 0 such that $x^{\prime}=[\alpha, x, z]$ and $y^{\prime}=[\alpha, y, z]$ both belong to $\Delta$. Since $R^{\prime}$ and $R^{\prime \prime}$ extend $R$ and are independent, it follows that $R^{\prime}$ and $R^{\prime \prime}$ rank $x$ and $y$ in the same way. Hence, $R^{\prime}=R^{\prime \prime}$.

Now, we focus on conditions that are strong enough to guarantee that a binary relation has an extension that is complete, transitive, and independent.

Let us insert here a result of Suzumura (1976, Thm 3) who solved a similar exercise. Suzumura started from a relation $R$ and looked for a complete and transitive relation $R^{*}$ such that $R \subset R^{*}$ and $P \subset P^{*}$. A natural way to proceed is to check whether the transitive closure $R_{T}$ of $R$ respects the asymmetric part, i.e. $P \subset P_{T}$. Apparently, this provides sufficient (and necessary) conditions: $R$ has an ordering extension if and only if

$$
\text { for each } x, y \text {, we have }(x, y) \in R_{T} \text { implies }(y, x) \notin P \text {. }
$$

Suzumura labeled this condition as consistency.

We proceed similarly. Let $R$ be a relation in $D$. The independent order relation $R^{*}$ in $D$ is said to be an independent ordering extension of $R$ if $R \subset R^{*}$ and $P \subset P^{*}$. The transitive and independent closure of $R$ is the smallest (for inclusion) relation in $D$ that includes $R$, satisfies transitivity and independence.

The next lemmas provide further insight in the transitive and independent closure of a relation in the hyperplane $H$. Let $R$ be a relation in $H$. Define the binary relation $R_{T I}$ in $H$ by $(x, y) \in R_{T I}$ if there exists a natural number $k$, elements $x_{1}=x, x_{2}, \ldots, x_{k+1}=y$ and $z_{1}, z_{2}, \ldots, z_{k}$ in $H$, and positive real numbers $\alpha_{1}, \alpha_{2}, \ldots, \alpha_{k}$ such that

$$
\text { for each } i=1,2, \ldots, k \text {, we have }\left(\left[\alpha_{i}, x_{i}, z_{i}\right],\left[\alpha_{i}, x_{i+1}, z_{i}\right]\right) \in R \text {. }
$$


Lemma 2. Let $R$ be a relation in $H$. The above defined relation $R_{T I}$ is its transitive and independent closure.

Proof. It is easy to see that $R_{T I}$ is transitive. To check independence, let $(x, y) \in R_{T I}$, $\beta \geq 0$, and let $q \in H$. We have to show that $\left(x^{\prime}=[\beta, x, q] ; y^{\prime}=[\beta, y, q]\right)$ belongs to $R_{T I}$. Since $(x, y) \in R_{T I}$, there exist elements $x_{i}\left(x_{1}=x\right.$ and $\left.x_{k+1}=y\right), z_{i}$ in $H$, and positive real numbers $\alpha_{i}$ such that

$$
\left(\left[\alpha_{i}, x_{i}, z_{i}\right],\left[\alpha_{i}, x_{i+1}, z_{i}\right]\right) \in R, \quad \text { for each } i=1,2, \ldots, k .
$$

Define $v_{i}=\frac{\left(1-\alpha_{i}\right) \beta z_{i}-(1-\beta) \alpha_{i} q}{\beta-\alpha_{i}}$ and $x_{i}^{\prime}=\left[\beta, x_{i}, q\right]$ in $H$. In case $\beta=\alpha_{i}$, the element $v_{i}$ has no role and can be chosen arbitrarily, e.g. put $v_{i}=0$. It follows that

$$
\left(\left[\frac{\alpha_{i}}{\beta}, x_{i}^{\prime}, v_{i}\right],\left[\frac{\alpha_{i}}{\beta}, x_{i+1}^{\prime}, v_{i}\right]\right) \in R, \quad \text { for each } i=1,2, \ldots, k \text {. }
$$

Hence, $\left(x^{\prime}, y^{\prime}\right) \in R_{T I}$.

Finally, we have to show that $R_{T I}$ is the smallest (for inclusion) independent and transitive relation containing $R$. Let $R^{\prime}$ be an independent and transitive extension of $R$. For each $(x, y)$ in $R_{T I}$, there exists elements $x_{1}=x, x_{2}, \ldots, x_{k+1}=y ; z_{1}, z_{2}, \ldots, z_{k}$ in $H$ and positive real numbers $\alpha_{1}, \alpha_{2}, \ldots, \alpha_{k}$ such that

$$
\left(\left[\alpha_{i}, x_{i}, z_{i}\right],\left[\alpha_{i}, x_{i+1}, z_{i}\right]\right) \in R, \quad \text { for each } i=1,2, \ldots, k .
$$

The independence of $R^{\prime}$ and the fact that $R \subset R^{\prime}$ imply that $\left(x_{i}, x_{i+1}\right) \in R^{\prime}$ for each $i=1,2, \ldots, k$. The transitivity of $R^{\prime}$ implies that $(x, y) \in R^{\prime}$. Therefore, $R_{T I} \subset R^{\prime}$.

Lemma 3. Let $R$ be a relation in $H$. Then, $(x, y)$ belongs to the transitive and independent closure $R_{T I}$ of $R$ if and only if

- either, $(x, y)$ belongs to the transitive closure of $R$;

- or, $x-y=\Sigma_{i=1}^{\ell} \beta_{i}\left(x_{i}-y_{i}\right)$, with $\left(x_{i}, y_{i}\right)$ in $R$ and $\beta_{i}>0$ for each $i$, and $\beta_{j} \neq 1$ for at least one $j$.

Proof. First, let $(x, y) \in R_{T I}$. The elements $x$ and $y$ are linked through a finite sequence $x=x_{1}, x_{2}, \ldots, x_{k+1}=y$; and there exist $z_{1}, z_{2}, \ldots, z_{k}$ in $H$ and $\alpha_{1}, \alpha_{2}, \ldots, \alpha_{k}>0$ such that

$$
\left(\left[\alpha_{i}, x_{i}, z_{i}\right],\left[\alpha_{i}, x_{i+1}, z_{i}\right]\right) \in R, \text { for each } i=1,2, \ldots, k .
$$

For each $i$ we obtain $\left[\alpha_{i}, x_{i}, z_{i}\right]-\left[\alpha_{i}, x_{i+1}, z_{i}\right]=\alpha_{i}\left(x_{i}-x_{i+1}\right)$. Multiply these equations by $1 / \alpha_{i}>0$, and add them up:

$$
x-y=x_{1}-x_{k+1}=\sum_{i=1}^{k} \frac{\left[\alpha_{i}, x_{i}, z_{i}\right]-\left[\alpha_{i}, x_{i+1}, z_{i}\right]}{\alpha_{i}} .
$$


In case $\alpha_{1}=\alpha_{2}=\cdots=\alpha_{k}=1$, then $(x, y)$ belongs to the transitive closure of $R$.

Next, assume $x-y=\Sigma_{i=1}^{\ell} \beta_{i}\left(x_{i}-y_{i}\right)$ with $\left(x_{i}, y_{i}\right)$ in $R, \beta_{i}>0$, and $\beta_{j} \neq 1$. We have to prove that $(x, y) \in R_{T I}$. We proceed by induction on $\ell$.

For $\ell=1$, it suffices to observe that the vector $z=\left(x-\beta_{1} x_{1}\right) /\left(1-\beta_{1}\right)$ in $H$ allows us to write $x=\left[\beta_{1}, x_{1}, z\right]$ and $y=\left[\beta_{1}, y_{1}, z\right]$.

Suppose the result holds up to $\ell$. Consider a positive linear combination of length $\ell+1$. Assume that $\beta_{1} \neq 1$. Consider $\left(x^{\prime}-y^{\prime}\right)=(1 / \beta) \times \Sigma_{i=2}^{\ell+1} \beta_{i}\left(x_{i}-y_{i}\right)$, with $0<\beta \neq 1$ such that at least one of the coefficients $\beta_{i} / \beta$ differs from 1 . From the induction basis, we obtain $\left(x^{\prime}, y^{\prime}\right) \in R_{T I}$. Hence, we can write

$$
x-y=\beta_{1}\left(x_{1}-y_{1}\right)+\beta\left(x^{\prime}-y^{\prime}\right), \quad \text { with } 0<\beta_{1} \neq 1 \text { and } 0<\beta \neq 1 .
$$

Let $z$ and $\tilde{y}$ in $H$ solve the equations $x_{1}=\left[1 / \beta_{1}, x, z\right]$ and $y_{1}=\left[1 / \beta_{1}, \tilde{y}, z\right]$. Independence implies $(x, \tilde{y}) \in R_{T I}$. Next, let $z^{\prime}$ and $y^{*}$ in $H$ solve the equations $x^{\prime}=\left[1 / \beta, \tilde{y}, z^{\prime}\right]$ and $y^{\prime}=\left[1 / \beta, y^{*}, z^{\prime}\right]$. Then, $\left(\tilde{y}, y^{*}\right) \in R_{T I}$. The transitivity of $R_{T I} \operatorname{implies}\left(x, y^{*}\right) \in R_{T I}$. Finally, the equations $x_{1}-y_{1}=(x-\tilde{y}) / \beta_{1}$ and $x^{\prime}-y^{\prime}=\left(\tilde{y}-y^{*}\right) / \beta$ imply that $y^{*}=y$.

Now, we are able to shift the result of Suzumura towards the present setting. We extend the definition of consistency and we state the main result of this section.

Definition 1. The relation $R$ in $D$ is said to be lottery-consistent if for each $x$ and $y$ in $D$, we have that $(x, y) \in R_{T I}$ implies $(y, x) \notin P$.

Theorem 1. Let $R$ be a relation in $\Delta$. Then, $R$ has an independent ordering extension in $\Delta$ if and only if $R$ is lottery-consistent.

Proof. Let $R^{*}$ be an independent ordering extension of $R$. Then, by Lemma $2, R \subset R_{T I} \subset$ $R^{*}$ and by the definition of extension $P \subset P^{*}$. Hence, it cannot happen that $(x, y) \in R_{T I}$ and $(y, x) \in P$; otherwise the combination $(x, y) \in R^{*}$ and $(y, x) \in P^{*}$ would occur. Conclude that $R$ is lottery-consistent.

The proof of the reverse implication is more involved. We indicate that the non-comparable part of an incomplete, independent, and transitive extension of $R$ can be further reduced by adding one single couple. We use this result, in combination with a free ultrafilter on an appropriate set, to define a complete, independent, and transitive extension of $R$.

Hence, let $R^{*}$ be an incomplete, independent, and transitive extension of $R$. Let $(x, y) \in$ $N^{*}$. Define the relation $Q=R^{*} \cup\{(x, y)\}$ and let $Q_{T I}$ be the transitive and independent closure of $Q$. We show that $Q_{T I}$ extends $R$. First, observe the inclusions $R \subset R^{*} \subset Q_{T I}$. The inclusion $P \subset P_{T I}$ is shown by contradiction. Therefore, assume the existence of a couple $(z, w)$ in $P$ such that $(w, z) \in Q_{T I}$. Apply Lemma 3 upon $Q_{T I}$ and obtain

$$
w-z=\sum_{i=1}^{\ell} \beta_{i}\left(x_{i}-y_{i}\right), \quad \text { with }\left(x_{i}, y_{i}\right) \in Q \text {, and } \beta_{i}>0 \text { for each } i \text {. }
$$

As $R^{*}$ extends $R$ and $(z, w) \in P \subset P^{*}$, the pair $(x, y)$ occurs at the right hand side; say $\left(x_{1}, y_{1}\right)=(x, y)$. Rewrite the previous equation:

$$
y-x=\gamma(z-w)+\Sigma_{i=2}^{\ell} \gamma_{i}\left(x_{i}-y_{i}\right), \quad \text { with } \gamma>0, \gamma_{i}>0 \text { for each } i .
$$


Lemma 3 implies that $R^{*}$ is able to compare $x$ and $y$. This conflicts with $(x, y) \in N^{*}$.

Next, let $[D]^{<\infty}$ be the collection of all finite subsets of $D$. For each $A$ in $[D]^{<\infty}$, let $S(A)$ collect all the finite supersets of $A$. For example, $S(\varnothing)=[D]^{<\infty}$. Let $\mathcal{U}$ be an ultrafilter that extends the filter generated by the family $\left\{S(A) \mid A \in[D]^{<\infty}\right\} .^{5}$

For each set $A \in[D]^{<\infty}$, let the relation $Q$ be an independent and transitive extension of $R$ that is able to compare all pairs in $A$. The relation $Q$ either coincides with $R_{T I}$ or can be obtained by adding a finite number of couples to $R_{T I}$ (as explained above). Denote the restriction of $Q$ to the set $A$ by $R_{A}$, i.e. $R_{A}=Q \cap(A \times A)$. Finally, define the relation $R^{*}$ in $D$ as follows:

$$
(x, y) \in R^{*} \quad \text { if and only if } \quad\left\{A \in[D]^{<\infty} \mid(x, y) \in R_{A}\right\} \in \mathcal{U} .
$$

We check that $R^{*}$ extends $R$, is independent and transitive, and complete.

(i) $R^{*}$ extends $R$. Let $R$ be able to compare $x$ and $y$. For each set $A$ in $S(\{x, y\})$ the relation $R_{A}$ agrees with $R$ on the pair $\{x, y\}$. As $S(\{x, y\})$ belongs to the ultrafilter $\mathcal{U}$, the relation $R^{*}$ extends $R$.

(ii) $R^{*}$ is transitive and independent. Let $x-y=\sum_{i=1}^{l} \alpha_{i}\left(x_{i}-y_{i}\right)$ with $\left(x_{i}, y_{i}\right) \in R^{*}$ and $\alpha_{i}>0$ for each $i=1,2, \ldots, l$. By definition, the sets $U_{i}=\left\{A \mid\left(x_{i}, y_{i}\right) \in R_{A}\right\}$ belong to $\mathcal{U}$. The finite intersection property implies that $U=U_{1} \cap U_{2} \cap \ldots \cap U_{l}$ and $U \cap S(\{x, y\})$ both belong to $\mathcal{U}$. Since each relation $R_{A}$ is transitive and independent, we have $(x, y) \in R_{A \cup\{x, y\}}$ for each $A$ in $U$. Hence, $(x, y) \in R^{*}$.

(iii) $R^{*}$ is complete. Consider the pair $\{x, y\}$. The collection $S(\{x, y\})$ splits up into three parts,

$$
S(\{x, y\})=\left\{A \mid(x, y) \in P_{A}\right\} \cup\left\{A \mid(y, x) \in P_{A}\right\} \cup\left\{A \mid(x, y),(y, x) \in R_{A}\right\} .
$$

Since $\mathcal{U}$ is an ultrafilter and $S(\{x, y\}) \in \mathcal{U}$, exactly one of these three parts belongs to $\mathcal{U}$. Conclude that $R^{*}$ is able to compare $x$ and $y$.

\section{Rationalizability of choice over lotteries}

This section extends Richter's result towards the rationalizability of individual choice over lotteries. At the end of this section we shortly discuss similar studies by Clark (1993) and by $\operatorname{Kim}(1996)$.

Consider the $(n-1)$-dimensional simplex $\Delta$ and let $\mathcal{S}$ be a collection of nonempty subsets of $\Delta$. A choice correspondence $C$ is a correspondence

$$
C: \mathcal{S} \longrightarrow \Delta: S \longmapsto C(S) \subset S .
$$

\footnotetext{
${ }^{5} \mathrm{~A}$ filter $\mathcal{F}$ on a set $\Omega$ is a subset of $2^{\Omega}$ that $(i)$ does not contain the empty set $(\varnothing \notin \mathcal{F}),(i i)$ satisfies the intersection property (if $A, B \in \mathcal{F}$, then $A \cap B \in \mathcal{F}$ ), and (iii) is closed for supersets (if $A \in \mathcal{F}$ and $A \subset B \subset \Omega$, then $B \in \mathcal{F}$ ). If the filter $\mathcal{F}$ contains, for each $A \subset \Omega$, either $A$ or its complement $\Omega-A$; then $\mathcal{F}$ is said to be an ultrafilter. An ultrafilter is a maximal (for inclusion) filter. Zorn's lemma implies that each filter extends to an ultrafilter. An ultrafilter that does not contain finite sets, is said to be free.
} 
The choice correspondence $C$ is said to be rationalizable if there exists an independent ordering $R^{*}$ in $\Delta$ such that for each $S$ in $\mathcal{S}$ the set $C(S)$ collects the maximizers of the restriction of $R^{*}$ to $S$, i.e.

for each $S$ in $\mathcal{S}: C(S)=M\left(R^{*} \mid S\right)=\left\{x \in S \mid\right.$ for each $y$ in $\left.S:(x, y) \in R^{*}\right\}$.

Observe that for a (rationalizable) choice correspondence the choice set $C(S)$ might be empty; e.g. if $S \subset \Delta$ is an open (in the Euclidean topology) set and if the ordering $R^{*}$ happens to be continuous, the set $M\left(R^{*} \mid S\right)$ of maximizers might be empty. As it is unclear what one should conclude on the basis of an empty choice set, we impose the choice correspondence to be decisive on $\mathcal{S}$, i.e. a set $S$ for which $C(S)=\varnothing$ is excluded from $\mathcal{S}$.

For a choice correspondence $C: \mathcal{S} \rightarrow \Delta$, the revealed preference relations $\tilde{R}$ and $\tilde{\pi}$ in $\Delta$ are defined as follows. The pair $(x, y)$ belongs to the revealed preference relation $\tilde{R}$ if and only if there is a set $S$ in $\mathcal{S}$ such that $x \in C(S)$ and $y \in S$. Furthermore, the pair $(x, y)$ belongs to the strict revealed preference relation $\tilde{\pi}$ if and only if there is a set $S$ in $\mathcal{S}$ such that $x \in C(S)$ while $y \in S \backslash C(S)$.

We extend the congruence axiom of Richter (1966).

Definition 2. A choice correspondence $C: \mathcal{S} \rightarrow \Delta$ is said to satisfy the congruence axiom if for each $x$ and $y$ in $\Delta$ we have

$$
(x, y) \in \tilde{R}_{T I} \quad \text { implies } \quad(y, x) \notin \tilde{\pi},
$$

where $\tilde{R}_{T I}$ is the transitive and independent closure of the revealed preference relation $\tilde{R}$.

We will show that this congruence axiom is strong enough to guarantee the choice correspondence to be rationalizable. The next lemma is a first step towards this result.

Lemma 4. If the choice correspondence $C: \mathcal{S} \rightarrow \Delta$ satisfies the congruence axiom, then the asymmetric part $\tilde{P}$ of the revealed preference relation $\tilde{R}$ coincides with the strict revealed preference relation $\tilde{\pi}$.

Proof. $(i): \tilde{P} \subset \tilde{\pi}$. If $(x, y) \in \tilde{P}$, then $(x, y) \in \tilde{R}$ and $(y, x) \notin \tilde{R}$. Hence, there exists a set $S$ such that $x \in C(S)$ and $y \in S$; and for each set $T$ containing $x$ and $y$, it holds that $y \notin C(T)$. Put $T=S$ and conclude that $x \in C(S)$ while $y \in S \backslash C(S)$, i.e. $(x, y) \in \tilde{\pi}$.

(ii) $: \tilde{\pi} \subset \tilde{P}$. If $(x, y) \in \tilde{\pi}$, then $(x, y) \in \tilde{R}$. In case also $(y, x) \in \tilde{R}$, the congruence axiom is violated: $(y, x) \in \tilde{R} \subset \tilde{R}_{T I}$ and $(x, y) \in \tilde{\pi}$. Therefore, $(y, x) \notin \tilde{R}$ and $(x, y) \in \tilde{P}$.

As a corollary we obtain that if a choice correspondence satisfies the congruence axiom, then the revealed preference relation is lottery-consistent. The main result of this section reads:

Theorem 2. Let the choice correspondence $C: \mathcal{S} \rightarrow \Delta$ be decisive on $\mathcal{S}$. Then, $C$ is rationalizable if and only if it satisfies the congruence axiom.

Proof. Let the independent ordering $R^{*}$ in $\Delta$ rationalize the choice correspondence $C$. Obviously, $R^{*}$ extends the revealed preference relation: $\tilde{R} \subset R^{*}$ and $\tilde{\pi} \subset P^{*}$. As $R^{*}$ is 
transitive and independent, $R^{*}$ includes the transitive and independent closure $\tilde{R}_{T I}$ of $\tilde{R}$ (use Lemma 2). Suppose now that $(y, x) \in \tilde{\pi}$. Then, $(y, x) \in P^{*}$ and $(x, y) \notin R^{*}$. As a consequence, if $(y, x) \in \tilde{\pi}$, then $(x, y) \notin \tilde{R}_{T I}$.

Let $C$ satisfy the congruence axiom. By Lemma 4, the revealed preference relation is lottery-consistent. Apply Theorem 1 and extend the revealed preference relation $\tilde{R}$ to an independent ordering $R^{*}$ in $\Delta$. Now, we have to verify whether $C(S)=M\left(R^{*} \mid S\right)$ holds each set $S$ in $\mathcal{S}$. Let $x \in C(S)$. Hence, for each $y$ in $S$ we have $(x, y) \in \tilde{R} \subset R^{*}$, i.e. $x \in M\left(R^{*} \mid S\right)$. Next, let $x \in S \backslash C(S)$. By assumption, $C$ is decisive on $S$ : there exists a $y$ in $S$ such that $y \in C(S)$. It follows that $(y, x) \in \tilde{\pi} \subset P^{*}$. Conclude that $x \notin M\left(R^{*} \mid S\right)$.

The ultimate goal is to establish a test for the null hypothesis

$$
H_{0} \text { : the individual choice correspondence } C: \mathcal{S} \longrightarrow \Delta \text { is rationalizable. }
$$

Of course, one can extract the binary relation behind the choice correspondence (by checking all the pairs in $\Delta$ ) and verify whether this relation is an independent ordering. In an empirical setting, however, this is impossible to manage. Theorem 2 allows us to test on the basis of a finite data set whether or not the null hypothesis should be rejected. As usual, not rejecting $H_{0}$ does not imply that $H_{0}$ is shown to hold. The next section returns to this issue.

Our approach to revealed preferences on lotteries differs from the work of Clark (1993) and Kim (1996). Where Kim (1996, Appendix) uses a generalization of the theorem of the alternative, we follow the axiomatic approach and start from the theory of binary extensions. Furthermore, Kim (1996, Thm 3.1) restricts the attention to finite choice sets. We do not impose restrictions on the size of the choice set. However, recall from Theorem 2 that we need the choice correspondence to be decisive on the choice sets.

Finally, as the revision of this paper was being completed, we learned of a result of Clark (1993). Although his Theorem 3 is very similar to our Theorem 2, there are some differences. First, Clark formulates different independence axioms. In the presence of transitivity and completeness, however, the combination of these axioms turn out to coincide with our independence condition. Second, Clark applies the Hausdorff maximality principle to obtain a complete relation. In contrast, we obtain completeness by means of a free ultrafilter (cf. Theorem 1). Hence, both proofs rely on non-constructive methods. The existence of a free ultrafilter, however, is a weaker assumption than the Hausdorff principle (which is equivalent to the axiom of choice). Third, we believe that our Lemma 3 provides additional insights to the concept of independency.

\section{Nash rationalization of collective choice}

Assume an experimental setting with individuals playing a game allowing mixtures over the set of pure strategies. The experimenter observes the mixtures selected by each individual separately. In case the profile of revealed preferences extends to a profile of independent 
orderings such that the selection corresponds to a Nash equilibrium, then we say that the observations support the hypothesis of Nash rationalizable behavior. If the data reject this hypothesis, then either some player does not consult a complete, transitive, and independent binary relation, or the Nash equilibrium is not the right equilibrium concept. This section develops such a test procedure.

We start by introducing some further notation. Let $J=\{1,2, \ldots, m\}$ be the set of players, $m \in \mathbb{N}$. Individual $j$ has $n_{j}$ pure strategies, his strategy space $\Delta_{j}$ is the $\left(n_{j}-1\right)$-dimensional simplex $\Delta^{n_{j}}$. A strategy profile is a vector $x=\left(x_{1}, x_{2}, \ldots, x_{m}\right)$ with $x_{j}$ in $\Delta_{j}$ the strategy of player $j$. The product set $\Delta_{J}=\Delta_{1} \times \Delta_{2} \times \cdots \times \Delta_{m}$ collects all the strategy profiles:

$$
\Delta_{J}=\left\{x=\left(x_{1}, x_{2}, \ldots, x_{m}\right) \mid x_{j}=\left(x_{j 1}, x_{j 2}, \ldots, x_{j n_{j}}\right) \in \Delta_{j}, j=1,2, \ldots, m\right\} .
$$

In order to distinguish the strategy $x_{j}$ in $\Delta_{j}$ of player $j$ from the strategies of his opponents, we denote the strategy profile $x$ also by $\left(x_{j}, x_{-j}\right)$ with $x_{-j}=\left(x_{1}, \ldots, x_{j-1}, x_{j+1}, \ldots, x_{k}\right)$ collecting the strategies of $j$ 's opponents.

A choice set $S$ is a cartesian product $S_{1} \times S_{2} \times \cdots \times S_{m} \subset \Delta_{J}$ with $S_{j}$ a nonempty subset of $\Delta_{j}$ for each $j$. A choice set represents an experiment in which players are confronted with restrictions within their strategy spaces. In the example in Section 1 (Table 1) each choice set is the convex hull of a finite set of points. The results below do not hinge on this convexity assumption.

For a choice set $S$, a strategy profile $x$ in $S$, and a player $j$ in $J$, we denote the cartesian product $S_{j} \times\left\{x_{-j}\right\}$ by $S_{j}^{x}$. In the choice set $S_{j}^{x}$ the strategy space of player $j$ is reduced to $S_{j}$ while the opponents only have one option (opponent $i$ selects $x_{i}$ from his strategy space $\left.\left\{x_{i}\right\}\right)$.

Let $\mathcal{S}$ be a collection of choice sets. A joint choice correspondence $C$ is a correspondence

$$
C: \mathcal{S} \longrightarrow \Delta_{J}: S \longmapsto C(S) \subset S \text {. }
$$

We assume that the choice correspondence $C$ is individually decisive, that is, we assume that $C\left(S_{j}^{x}\right)$ is nonempty for each choice set in $\mathcal{S}$ of the form $S_{j}^{x}$. In words, when the choice of all but one players is limited to only one option, then this one player should be able to select a strategy. ${ }^{6}$

In order to employ individual decisiveness, we impose that for each choice set $S$ in $\mathcal{S}$ all one-person choice sets $S_{j}^{x}$ derived from $S$ also belong to $\mathcal{S}$; Sprumont (2000) and Galambos (2005) impose the same condition.

In contrast to the previous section, we do not equip the players with a preference relation on the set $\Delta_{J}$ of strategy profiles. Instead, we assume that the players have preferences over the probability distributions of pure strategy profiles (e.g. via the payoffs corresponding to the pure strategies). As each player $j$ has $n_{j}$ pure strategies, these pure strategies generate

\footnotetext{
${ }^{6}$ Decisiveness is usually assumed in this context. Clark (1995) discusses indecisive choice functions.
} 
$n=n_{1} n_{2} \cdots n_{m}$ pure strategy profiles. ${ }^{7}$ The $(n-1)$-dimensional simplex $\Delta^{n}$ collects all the distributions over these profiles. Let $d$ denote the map that converts a strategy profile in $\Delta_{J}$ into a probability distribution in $\Delta^{n}$ :

$$
d: \Delta_{J} \longrightarrow \Delta^{n}: x \longmapsto d(x), \text { with } d_{i_{1}, i_{2}, \ldots, i_{m}}(x)=x_{1 i_{1}} x_{2 i_{2}} \cdots x_{m i_{m}},
$$

where $i_{j}$ runs over the pure strategies 1 to $n_{j}$ of player $j$. Within this notation, we can define Nash rationalizability of choice over lotteries.

Definition 3. The joint choice correspondence $C: \mathcal{S} \rightarrow \Delta_{J}$ is said to be Nash rationalizable if there exists a profile $\left(R_{1}^{*}, R_{2}^{*}, \ldots, R_{m}^{*}\right)$ of independent orderings in $\Delta^{n}$ such that for each $S$ in $\mathcal{S}$, we have

$$
x \in C(S) \text { if and only if } d(x) \in M\left(R_{j}^{*} \mid d\left(S_{j}^{x}\right)\right) \text { for each } j \text { in } J .
$$

In words, a joint choice correspondence is Nash rationalizable if each player consults an independent ordering to select his own strategy conditional upon his opponents' strategies.

For a Nash rationalizable choice correspondence it holds that, whenever $S_{j}^{x}$ is in the domain $\mathcal{S}, x \in C\left(S_{i}^{x}\right)$ if and only if $d(x) \in M\left(R_{i}^{*} \mid d\left(S_{i}^{x}\right)\right)$. Hence, if $C$ is Nash rationalizable and $S_{j}^{x} \in \mathcal{S}$ for each $j$ in $J$, then $x \in C(S)$ if and only if $x \in C\left(S_{j}^{x}\right)$ for each $j$ in $J$. The noncooperative behavior of the players is clearly incorporated in the definition of Nash rationalizability: a joint strategy is chosen if no single player has an incentive to deviate.

We modify the definitions of the revealed preference relations from the previous section towards the present setting. Let $a, b \in \Delta^{n}$.

We start with the revealed preference relations $\tilde{R}_{1}, \tilde{R}_{2}, \ldots, \tilde{R}_{m}$. We have $(a, b) \in \tilde{R}_{j}$ if there exist an $x$ in $\Delta_{J}$ and an $S_{j}^{x}$ in $\mathcal{S}$ such that $y \in S_{j}^{x}, x \in C\left(S_{j}^{x}\right)$, and $(a, b)=(d(x), d(y))$.

Next, we consider the strict revealed preference relations $\tilde{\pi}_{1}, \tilde{\pi}_{2}, \ldots, \tilde{\pi}_{m}$. We have $(a, b) \in \tilde{\pi}_{j}$ if there exist an $x$ in $\Delta_{J}, S_{j}^{x}$ in $\mathcal{S}$, and $y$ in $S_{j}^{x}$ such that $x \in C\left(S_{j}^{x}\right), y \in S_{j}^{x} \backslash C\left(S_{j}^{x}\right)$, and $(a, b)=(d(x), d(y))$.

These modifications imply that a player is only able to reveal preferences conditional upon a status quo of his opponents' strategies. A player is able to select $a$ above $b$ only if he has $a$ and $b$ at 'his' disposal, i.e. only if he is able to switch between $a$ and $b$ without the cooperation of any other player.

Similar to the previous section, we search for conditions upon the revealed preferences to guarantee the Nash rationalizability of a choice correspondence $C: \mathcal{S} \rightarrow \Delta_{J}$.

Definition 4. The joint choice correspondence $C$ is said to satisfy the joint congruence axiom if for all $a$ and $b$ in $\Delta^{n}$ and for each $j$ in $J$, we have

$$
(a, b) \in \tilde{R}_{j T I} \quad \text { implies } \quad(b, a) \notin \tilde{\pi}_{j}
$$

with $\tilde{R}_{j T I}$ the transitive and independent closure of the revealed preference relation $\tilde{R}_{j}$.

\footnotetext{
${ }^{7}$ The example in Section 1 exhibits four pure strategy profiles: $(U, L),(U, R),(D, L)$, and $(D, R)$.
} 
The next lemma states that if a joint choice correspondence satisfies the joint congruence axiom, then the revealed preference relations are lottery-consistent. Its proof only involves minor modifications of the proof of Lemma 4 and is omitted.

Lemma 5. If $C$ satisfies the joint congruence axiom, then for each player $j$ the asymmetric part $\tilde{P}_{j}$ of the revealed preference relation $\tilde{R}_{j}$ coincides with the strict revealed preference relation $\tilde{\pi}_{j}$.

At this point we are ready to provide conditions for the rationalizability of the individual choice correspondence $S_{j}^{x} \mapsto C\left(S_{j}^{x}\right)$. In order to obtain rationalizability of the joint choice correspondence $S \rightarrow \Delta_{J}$, we need some 'local-global' condition to link the collective choice from a set $S$ with the individual choices from the sets $S_{j}^{x}$. Here, we return to the noncooperative nature of the Nash equilibrium.

Definition 5. The joint choice correspondence $C: \mathcal{S} \rightarrow \Delta_{J}$ is said to be noncooperative if for each $S$ in $\mathcal{S}$ we have

$$
x \in C(S) \text { if and only if } \quad x \in C\left(S_{j}^{x}\right) \text { for each } j \text { in } J .
$$

In words, if a strategy profile $x$ is selected from $S$ then each player $j$ selects this profile when the choice set $S$ contracts or shrinks into his individual choice set $S_{j}^{x}$. And, if the group of players jointly select $x$ from the choice sets $S_{j}^{x}$, then the group of players jointly select $x$ from the union $S=S_{1}^{x} \cup S_{2}^{x} \cup \ldots \cup S_{m}^{x}$. As such, this axiom has some flavor of a contraction-expansion property.

Noncooperation might be observed even in those cases where the individuals do not select a Nash equilibrium or do not consult an ordering. While choice sets of the type $S_{j}^{x}$ are sufficient to test the joint congruence axiom, different types of choice sets are involved to test the local-global condition of noncooperation. Section 6 provides further evidence for the independency of these two axioms: we provide data sets that support one axiom and violate the other axiom. Furthermore, the axioms of joint congruence and noncooperation only depend on the choice sets and the selections made from these sets. As such, these axioms are testable. The combination of noncooperation and the joint congruence axiom implies the rationalizability of the joint choice correspondence.

Theorem 3. Let the joint choice correspondence $C: \mathcal{S} \rightarrow \Delta_{J}$ be individually decisive and assume that $\mathcal{S}$ satisfies the domain condition. Then, $C$ is Nash rationalizable if and only if $C$ is noncooperative and satisfies the joint congruence axiom.

Proof. Let $C$ be Nash rationalizable through the profile $\left(R_{1}^{*}, R_{2}^{*}, \ldots, R_{m}^{*}\right)$ of independent orderings in $\Delta^{n}$. To prove that $C$ satisfies the joint congruence axiom, one can apply Theorem 2 upon the individual choice correspondences $C: \mathcal{S}_{j} \rightarrow \Delta_{j}$, where $\mathcal{S}_{j}$ collects all the choice sets of the form $S_{j}^{x}$ with $S$ running through the collection $\mathcal{S}$. That $C$ is noncooperative has been argued above (see Definition 4 ).

Now, suppose that $C$ is noncooperative and satisfies the joint congruence axiom. Then, each revealed preference relation $\tilde{R}_{j}$ is lottery-consistent and extends to an independent 
ordering $R_{j}^{*}$ in $\Delta^{n}$ (use Theorem 2). We have to check whether for each $S$ in $\mathcal{S}$, for each $x$ in $S$, it holds that

$$
x \in C(S) \text { if and only if } d(x) \in M\left(R^{*} \mid d\left(S_{j}^{x}\right)\right) \text { for each } j \text { in } J .
$$

Let $x \in C(S)$. As $C$ is noncooperative, it follows that $x \in C\left(S_{j}^{x}\right)$ for each $j$ in $J$. Hence, for each $y$ in $S_{j}^{x}$ we have $(d(x), d(y)) \in \tilde{R}_{j} \subset R_{j}^{*}$. It follows that $d(x) \in M\left(R_{j}^{*} \mid S_{j}^{x}\right)$ for each $j$ in $J$.

Finally, let $x \in S \backslash C(S)$ and assume that $d(x) \in d(S)$. As $C$ is noncooperative, there exists at least one player $j$ for which $x \notin C\left(S_{j}^{x}\right)$. Since $C$ is individually decisive, there exists a $y$ in $S_{j}^{x}$ such that $y \in C\left(S_{j}^{x}\right)$. Therefore $(d(y), d(x)) \in \tilde{\pi}_{j} \subset P_{j}^{*}$. It follows that for player $j$ we have that $d(x) \notin M\left(R_{j}^{*} \mid d\left(S_{i}^{x}\right)\right)$.

This theorem establishes a rule to judge whether or not the hypothesis

$H_{0}$ : the collective choice correspondence $C: \mathcal{S} \longrightarrow \Delta_{J}$ is Nash rationalizable

should be rejected. The test is exact in the sense that as soon as the observations conflict with the axiom of congruence, the null hypothesis is false with certainty. The probability to reject the hypothesis when it is actually true is zero. Let us apply the test upon the data (Table 1) presented in Section 1.

Denote $x=C(S), x^{\prime}=C\left(S^{\prime}\right)$, and $x^{\prime \prime}=C\left(S^{\prime \prime}\right)$. Let us list the four pure strategy profiles: $(U, L),(U, R),(D, L)$, and $(D, R)$. We have that $d(x)=(0.12,0.28,0.18,0.42)$.

Use the axiom of noncooperation to conclude that player 1 reveals to (weakly) prefer $(0.4,0.6)$ above any other strategy available to him, such as $(0.3,0.7)$. Let us write $y=$ $(0.3,0.7) \times(0.3,0.7)$, and $d(y)=(0.09,0.21,0.21,0.49)$. As such we learn that $(d(x), d(y)) \in$ $\tilde{R}_{1 T I}$.

Similarly, $d\left(x^{\prime}\right)=(0.168,0.232,0.252,0.348)$. Since also the strategy $(0.42,0.58)$ is at the disposal of player 1, it follows (again, use the axiom of noncooperation) that $\left(d\left(x^{\prime}\right), d\left(y^{\prime}\right)\right) \in$ $\tilde{R}_{1 T I}$, with $d\left(y^{\prime}\right)=(0.2205,0.3045,0.1995,0.2755) \in \Delta^{4}$.

Finally, $d\left(x^{\prime \prime}\right)=(0.25,0.25,0.25,0.25)$. The available strategy $(0.2,0.8)$ leads to the distribution $d\left(y^{\prime \prime}\right)=(0.1,0.1,0.4,0.4)$. The data imply $\left(d\left(x^{\prime \prime}\right), d\left(y^{\prime \prime}\right)\right) \in \tilde{\pi}_{1}$.

One can check that $2(d(x)-d(y))+4\left(d\left(x^{\prime}\right)-d\left(y^{\prime}\right)\right)+\left(d\left(x^{\prime \prime}\right)-d\left(y^{\prime \prime}\right)\right)=0$. Solve this equation for $d\left(y^{\prime \prime}\right)-d\left(x^{\prime \prime}\right)$ and conclude (use Lemma 3) that $\left(d\left(y^{\prime \prime}\right), d\left(x^{\prime \prime}\right)\right)$ belongs to the independent and transitive closure of $\tilde{R}_{1}$. This contradicts our extended version of Richter's congruence axiom. Therefore, the data reject the hypothesis $H_{0}$.

We close this section with a discussion of work by Galambos (2005), who obtains a single condition - labeled I-congruence - for the Nash rationalizability in pure strategies. Define the binary relation $R_{j}^{*}$ in the product strategy space $\Delta_{J}$ by $(x, y) \in R_{j}^{*}$ if $(i)$ the strategies $x_{i}$ and $y_{i}$ coincide for each $i \neq j$ and $(i i)$ there exists an $S$ in $\mathcal{S}$ for which $x \in C(S)$. In the assumption that the axiom of noncooperation holds, the relations $R_{j}^{*}$ and $\tilde{R}_{j}$ coincide. 
Let $R_{j T I}^{*}$ be the transitive and independent closure of $R_{j}^{*}$. We rephrase the I-congruence axiom as follows. For each choice set $S$ and for each strategy profile $x$,

$$
\text { if }(x, y) \in R_{j T I}^{*} \text { for each } y \text { in } S_{j}^{x} \text { and each } j \text { in } N \text {, then } x \in C(S) \text {. }
$$

This I-congruence condition combines the axioms of joint congruence and noncooperation and therefore provides an alternative rationalizability condition. The above Theorem 3 uses two axioms that represent two separate ideas. The axiom of joint congruence reflects the idea that each individual, independently of the behavior of his opponents, consults an independent preference relation. The axiom of noncooperation reflects the idea of the Nash equilibrium that each individual takes the behavior of the opponents as given.

\section{Persistence axioms of Sprumont}

In this section we show that the persistence conditions of Sprumont are equivalent to our conditions for Nash rationalizability (when restricted to the setting of pure strategies). As such we indicate that our Theorem 3 extends Theorem 2 of Sprumont (2000) to cases involving mixtures over pure strategies.

Let $A_{j}$ be the set of pure strategies available to player $j$ and let $A=A_{1} \times A_{2} \times \cdots \times A_{m}$ be the set of all joint pure strategies. When restricted to the pure strategies, the map $d$ from the space $\Delta_{J}$ of strategy profiles to the space $d\left(\Delta^{n}\right)$ of distributions over the pure strategy profiles remains one-to-one. Observing a (degenerate) distribution in $\Delta^{n}$ boils down to observing the pure strategies selected by the players.

Sprumont (2000) considers the collection $\mathcal{S}$ of cartesian products $S_{1} \times S_{2} \times \cdots \times S_{m}$ with $\varnothing \neq S_{j} \subset A_{j}$ and studies joint choice correspondences $C: \mathcal{S} \rightarrow A$ that are decisive on $\mathcal{S}$. Such a correspondence $C$ is said to be persistent under expansion if for each $S$ and $T$ in $\mathcal{S}$ it holds that $C(S) \cap C(T) \subset C(S \vee T)$, with $S \vee T$ the smallest choice set in $\mathcal{S}$ that includes $S$ and $T$.

Furthermore, $C$ is said to be persistent under contraction if $(i)$ for each $S$ and $T$ in $\mathcal{S}$ with $T \subset S$ it holds that $C(S) \cap T \subset C(T)$ and (ii) for each $S$ and $T$ in $\mathcal{S}$ with $T \subset S_{j}^{x}$ and $C\left(S_{j}^{x}\right) \cap T \neq \varnothing$, it holds that $C(T) \subset C\left(S_{j}^{x}\right)$.

The next proposition phrases the equivalence between the two approaches. Of course, this proposition mutually supports our results and those of Sprumont.

Proposition. Let $C: \mathcal{S} \rightarrow A$ be a decisive joint choice correspondence. Then, $C$ is noncooperative and satisfies the joint congruence axiom (taking only the transitive closure into account) if and only if $C$ is persistent under expansion and persistent under contraction.

Proof. First, assume $C$ is noncooperative and satisfies the congruence axiom. Let us check whether $C$ is persistent under expansion. Let $S$ and $T$ in $\mathcal{S}$. If $a \in C(S) \cap C(T)$, then (use noncooperation) $a \in C\left(S_{j}^{a}\right) \cap C\left(T_{j}^{a}\right)$ for each $j$ in $J$. Hence, the players reveal $(a, b) \in \tilde{R}_{j}$ for each $b$ in $S_{j}^{a} \cup T_{j}^{a}$. If for player $i$ in $J$ we have $a \notin C\left((S \vee T)_{i}^{a}\right)$, then this player reveals to 
strictly prefer some action $b$ (the decisiveness of $C$ implies the existence of such an action) over $a$, i.e. $(b, a) \in \tilde{\pi}_{i}$. This contradicts the congruence axiom. Hence, $a \in C\left((S \vee T)_{j}^{a}\right)$ for each $j$ in $J$. Noncooperation implies $a \in C(S \vee T)$.

We now verify persistence under contraction. Condition $(i)$. Let $T \subset S$ and $a \in C(S) \cap T$. Noncooperation implies that each player $j$ selects $a$ from the individual choice set $S_{j}^{a}$. The congruence axiom implies that each player $j$ selects $a$ from the smaller choice set $T_{j}^{a}$. Conclude that $a \in C(T)$.

Contraction condition (ii). Let $T \subset S_{j}^{x}, b \in C\left(S_{j}^{x}\right) \cap T$, and $a \in C(T)$. As a consequence, $(a, b) \in \tilde{R}_{j}$. Hence, if this player does not select $a$ from $S_{j}^{x}$, there exists a $d$ in $S_{j}^{x}$ such that $(d, a) \in \tilde{\pi}_{j}$. As $b \in C\left(S_{j}^{x}\right)$ and $d \in S_{j}^{x}$, it follows that $(b, d) \in \tilde{R}_{j}$. These observations contradict the congruence axiom: $(a, d)$ belongs to the transitive closure of $\tilde{R}_{j}$, while $(d, a) \in \tilde{\pi}_{j}$.

Next, suppose that $C$ satisfies the persistence axioms. Let us check the congruence axiom. Hence, assume $(a, b)$ belongs to the transitive closure of $\tilde{R}_{j}$ with $j$ in $J$. Denote the sequence from $a$ to $b$ by $a=a_{1}, a_{2}, \ldots, a_{k+1}=b$, i.e. we have $\left(a_{1}, a_{2}\right),\left(a_{2}, a_{3}\right), \ldots,\left(a_{k}, a_{k+1}\right) \in \tilde{R}_{j}$. As player $j$ is only able to reveal preferences conditional upon a status quo of his opponents, it must be the case that $a_{1}, a_{2}, \ldots, a_{k+1} \in A_{j} \times\left\{a_{-j}\right\}$, remember that $a_{-j}$ collects the strategies of $j$ 's opponents. Persistence under contraction (part $i$ ) allows us to focus on the sets $S_{\ell}=\left\{a_{1}, a_{2}, \ldots, a_{\ell}\right\}$ with $\ell=2,3, \ldots, k+1$. One can check that $C\left(S_{\ell}\right) \cap S_{\ell-1} \neq \varnothing$. From persistence under contraction (part ii) it follows that $C\left(S_{\ell-1}\right) \subset C\left(S_{\ell}\right)$. Therefore, $a \in C\left(S_{k+1}\right)$, and $a \in C(\{a, b\})$. Conclude that $(b, a) \notin \tilde{\pi}_{j}$ and $(a, b) \notin \tilde{R}_{j}$.

Finally, we check for noncooperation. Let $x \in C\left(S_{j}^{x}\right)$ for each $j$ in $J$. Persistence under expansion implies $x \in C\left(S_{1}^{x} \vee S_{2}^{x} \vee \ldots \vee S_{m}^{x}\right)=C(S)$. And, if $x \in C(S)$, then $x \in C\left(S_{j}^{x}\right)$ for each $j$ (use persistence under contraction).

\section{Noncooperation versus joint congruence}

We show that noncooperation and joint congruence are independent axioms. We provide two examples. The first observed data set supports noncooperation and conflicts with joint congruence. The second data set conflicts with noncooperation and supports the joint congruence axiom.

Example 1 (noncooperation but not joint congruence).

Consider a setup with two individuals. Each individual has two pure strategies, a mixed strategy by player $j$ is denoted by $x_{j}=\left(x_{j 1}, x_{j 2}\right)$ with $x_{j 1}+x_{j 2}=1, j=1,2$.

The following data (only singleton-selections are involved) are observed:

\begin{tabular}{|c|c|c|}
\hline choice set & & observed selection \\
\hline $\begin{array}{l}A=\left\{\left(x_{1}, x_{2}\right) \mid 1 \geq x_{11} \geq 0.5,\right. \\
B=\left\{\left(x_{1}, x_{2}\right) \mid 0.5 \geq x_{11} \geq 0,\right.\end{array}$ & $\begin{array}{l}\left.x_{21}=0.3\right\} \\
\left.x_{21}=0.4\right\}\end{array}$ & $\begin{array}{l}C(A)=z=((0.5,0.5) ;(0.3,0.7)) \\
C(B)=y=((0.5,0.5) ;(0.4,0.6))\end{array}$ \\
\hline$D=\left\{\left(x_{1}, x_{2}\right) \mid 1 \geq x_{11} \geq 0.5\right.$, & $\left.1 \geq x_{21} \geq 0.5\right\}$ & $C(D)=w=((0.5,0.5) ;(0.5,0.5))$ \\
\hline
\end{tabular}

In addition, we observe $C\left(A_{j}^{z}\right)=z, C\left(B_{j}^{y}\right)=y$, and $C\left(D_{j}^{w}\right)=w$ where $j=1,2$. Hence, 
the axiom of noncooperation is supported. We now argue that the data do violate joint congruence. Let $\left\{z^{\prime}\right\}=\{((0.6,0.4),(0.3,0.7))\} \in A_{1}^{z},\left\{y^{\prime}\right\}=\{(0.4,0.6),(0.4,0.6)\} \in B_{1}^{y}$, and $\left.\left\{w^{\prime}\right\}=\{(0.6,0.4),(0.5,0.5))\right\} \in D_{1}^{w}$.

We have $\left(d(z), d\left(z^{\prime}\right)\right) \in \tilde{\pi}_{1},\left(d(y), d\left(y^{\prime}\right)\right) \in \tilde{R}_{1}$, and $\left(d(w), d\left(w^{\prime}\right)\right) \in \tilde{R}_{1}$. The equality

$$
d\left(z^{\prime}\right)-d(z)=2\left[d(y)-d\left(y^{\prime}\right)\right]+\left[d(w)-d\left(w^{\prime}\right)\right]
$$

implies that $\left(d\left(z^{\prime}\right), d(z)\right)$ belongs to $\tilde{R}_{1 T I}$. Hence, joint congruence is violated.

Example 2 (joint congruence but not noncooperation).

We keep the setting and the notation of the previous example. We adjust the set $D$ and the selection $C(D)$ :

\begin{tabular}{|c|c|c|}
\hline choice set & & observed selection \\
\hline $\begin{array}{l}A=\left\{\left(x_{1}, x_{2}\right) \mid 1 \geq x_{11} \geq 0.5,\right. \\
B=\left\{\left(x_{1}, x_{2}\right) \mid 0.5 \geq x_{11} \geq 0,\right.\end{array}$ & $\begin{array}{l}\left.x_{21}=0.3\right\} \\
\left.x_{21}=0.4\right\}\end{array}$ & $\begin{array}{l}C(A)=z=((0.5,0.5) ;(0.3,0.7)) \\
C(B)=y=((0.5,0.5) ;(0.4,0.6))\end{array}$ \\
\hline$D=\left\{\left(x_{1}, x_{2}\right) \mid 0.5 \geq x_{11} \geq 0\right.$, & $\left.1 \geq x_{21} \geq 0.5\right\}$ & $C(D)=w=((0,1) ;(0.5,0.5))$ \\
\hline
\end{tabular}

Furthermore, $C\left(A_{1}^{z}\right)=C\left(A_{2}^{z}\right)=z, C\left(B_{1}^{y}\right)=C\left(B_{2}^{y}\right)=y$, and $C\left(D_{2}^{w}\right)=w$. Finally, the selection $C\left(D_{1}^{w}\right)=\{((0.5,0.5),(0.5,0.5)\}$ and differs from $C(D)$. Hence, noncooperation is violated. In order to check the joint congruence axiom, we consider the revealed preference $\tilde{R}_{1}$ and strict revealed preference $\tilde{\pi}_{1}$ of individual 1 . For example

$$
\left(d(z), d\left(z^{\prime}\right)\right) \in \tilde{R}_{1} \text { as soon } z^{\prime}=\left(\left(z_{1}^{\prime}, 1-z_{1}^{\prime}\right),(0.3,0.7)\right) \text { and } 1 \geq z_{1}^{\prime} \geq 0.5,
$$

and

$$
\left(d(z), d\left(z^{\prime}\right)\right) \in \tilde{\pi}_{1} \text { as soon } z^{\prime}=\left(\left(z_{1}^{\prime}, 1-z_{1}^{\prime}\right),(0.3,0.7)\right) \text { and } 1 \geq z_{1}^{\prime}>0.5 .
$$

We proceed by contradiction and assume that $\tilde{R}_{1}$ and $\tilde{\pi}_{1}$ do not satisfy the axiom of joint congruence. Then, there exist finite subsets $A^{\prime} \subset A, B^{\prime} \subset B$, and $D^{\prime} \subset D$ and corresponding vectors $\alpha, \beta$, and $\gamma$ of positive real numbers such that

$$
\sum_{z^{i} \in A^{\prime}} \alpha_{i}\left(d(z)-d\left(z^{i}\right)\right)+\sum_{y^{j} \in B^{\prime}} \beta_{j}\left(d(y)-d\left(y^{j}\right)\right)+\sum_{w^{k} \in D^{\prime}} \gamma_{k}\left(d(w)-d\left(w^{k}\right)\right)=0,
$$

where at least one element in $A^{\prime} \cup B^{\prime} \cup C^{\prime}$ is strictly preferred over the corresponding selection. As both players have two pure strategies, equation (3) lives in $\mathbb{R}^{4}$. We consider the projection generated by strategy 1 for both players, and the projection generated by strategy 1 for player 1 and strategy 2 for player 2 :

$$
\begin{aligned}
& \sum_{i} \alpha_{i}\left(0.5-z_{1}^{i}\right) 0.3+\sum_{j} \beta_{j}\left(0.5-y_{1}^{j}\right) 0.4+\sum_{k} \gamma_{k}\left(0.5-w_{1}^{k}\right) 0.5=0 \\
& \sum_{i} \alpha_{i}\left(0.5-z_{1}^{i}\right) 0.7+\sum_{j} \beta_{j}\left(0.5-y_{1}^{j}\right) 0.6+\sum_{k} \gamma_{k}\left(0.5-w_{1}^{k}\right) 0.5=0 .
\end{aligned}
$$


Multiply equation (4) by two and subtract equation (5) and obtain:

$$
-\sum_{i} \alpha_{i}\left(0.5-z_{1}^{i}\right) 0.1+\sum_{j} \beta_{j}\left(0.5-y_{1}^{j}\right) 0.2+\sum_{k} \gamma_{k}\left(0.5-w_{1}^{k}\right) 0.5=0 .
$$

Since $z_{1}^{i} \geq 0.5$, each term in the previous equation is nonnegative and should be equal to zero. We obtain a contradiction. The joint congruence condition is satisfied for individual 1. The congruence condition for individual 2 is checked for in a similar way.

\section{References}

Arrow KJ (1959) Rational choice functions and orderings. Economica $26: 121-127$.

Clark SA (1993) Revealed preference and linear utility. Theory and Decision 34:21-45.

Clark SA (1995) Indecisive choice theory. Mathematical Social Sciences 30(2) : 155-170.

Clark SA (2000) Revealed preference and expected utility. Theory and Decision 49:159-174.

Conlisk J (1989) Three variants on the Allais example. American Economic Review 79(3) : 392-407.

Galambos Á (2005) Revealed preference in game theory. Working paper, Kellogg School of Management, Northwestern University. http://www.cerge-ei.cz/pdf/events/papers/060130_t.pdf

Kim T (1996) Revealed preference theory on the choice of lotteries. Journal of Mathematical Economics $26(4): 463-477$.

MacDonald DN, Wall JL (1989) An experimental study of the Allais paradox over losses: some preliminary evidence. Quarterly Journal of Business and Economics 28(4) : 43-60.

Myerson RB (1997) Game theory, analysis of conflict. Harvard University Press, Cambridge.

Oliver A (2003) A quantitative and qualitative test of the Allais paradox using health outcomes. Journal of Economic Psychology 24(1) : 35-48.

Ray I, Zhou L (2001) Game theory via revealed preferences. Games and Economic Behavior 37(2) : 415424 .

Richter MK (1966) Revealed preference theory. Econometrica 34(3):635-645.

Sen AK (1971) Choice functions and revealed preferences. Review of Economic Studies 38:307-317.

Shachat JM (2002) Mixed strategy play and the minimax hypothesis. Journal of Economic Theory 104: $189-226$.

Sopher B, Narramore M (2000) Stochastic choice and consistency in decision making under risk: an experimental study. Theory and Decision $48: 323-350$.

Sprumont Y (2000) On the testable implications of collective choice theories. Journal of Economic Theory $93: 205-232$.

Suzumura K (1976) Remarks on the theory of collective choice. Economica 43 : 381-390. 